\title{
ESTUDO DA DINÂMICA DA LUTA E A INFLUÊNCIA DO GOLDEN SCORE NA PREPARAÇÃO FÍSICA DE JUDOCAS DE ALTO NÍVEL
}

Rodrigo Ribeiro Rosa

Fabrício Boscolo Del Vecchio

Leonardo José Mataruna dos Santos

Mara Patrícia Traina Chacon-Mikahil

Paulo Roberto de Oliveira

\section{Resumo}

As novas tendências do treinamento desportivo aplicado ao judô propõem observações dos esforços competitivos, destacando o advento do Golden Score (GS), que altera o tempo possível de combate. Por isso, foram observados 23 judocas brasileiros do sexo masculino, em 42 lutas realizadas na $1^{\text {a }}$ Seletiva Nacional de 2003, evento que formou a base da Seleção Brasileira de Judô do citado ano. A análise das variáveis da dinâmica de combate não mostrou diferenças significativas, evidenciando que a inclusão do GS não alterou a dinâmica da luta, porém, devido ao observado aumento do volume do tempo de luta referente ao GS, apontam para uma necessária alteração dos conteúdos das cargas específicas de treino, buscando otimizar o rendimento desportivo nessa nova realidade competitiva.

\section{Palavras-Chave}

Judô; Treinamento desportivo; Resistência; Força.

\section{STUDY OF THE DYNAMICS OF THE FIGHT AND THE INFLUENCE OF THE GOLDEN SCORE IN THE PHYSICAL PREPARATION OF HIGH LEVEL JUDOKAS}

Rodrigo Ribeiro Rosa

Fabrício Boscolo Del Vecchio

Leonardo José Mataruna dos Santos

Mara Patrícia Traina Chacon-Mikahil

Paulo Roberto de Oliveira

\begin{abstract}
The new trends of the applied sports training to judo consider comments of the competitive efforts, detaching the advent of Golden Score (GS) that it modifies the possible time of combat. Therefore, had been observed 23 Brazilian judokas of the masculine sex, in 42 fights carried through in $1^{\text {a }}$ National Selective, event that formed the base of the 2003 Brazilian Election of Judo. The analysis of the variable of the combat dynamics did not show significant differences, evidencing that the inclusion of the GS did not modify the dynamics of the fight, however, the increase of the fight time, which had to the GS, point with respect to a necessary alteration of the contents of specific loads of trainings, searching to rationally optimize the sporting income in this new joined reality in the competition.
\end{abstract}

\section{Key-Words}

Judo; Sports training; Endurance; Strength. 


\section{Resumen}

Las nuevas tendencias del entrenamiento deportivo aplicado al judo consideran los comentarios de los esfuerzos competitivos, separando el advenimiento de la Tecnica de Oro (TO), que modifica la época posible del combate. Por lo tanto, habían sido los 23 judokas brasileños observados del sexo masculino, en 42 luchas llevadas a través en $1^{\circ}$ selectivo nacional, el acontecimiento que formó la base de la selección brasileña de Judo en 2003. El análisis de la variable de la dinámica del combate no demostró las diferencias significativas, evidenciando que la inclusión de la TO no modificó la dinámica de la lucha, sin embargo, el aumento del tiempo de la lucha, que tuvo que la TO, punto con respecto a una alteración necesaria del contenido de las cargas específicas de los entrenamientos, buscando la optimización racional del rendimiento deportivo en esta nueva realidad unida en la competición.

\section{Palabras-Clave}

Judo; Entrenamiento deportivo; Resistencia; Fuerza. 


\section{INTRODUÇÃO}

$\mathrm{O}$ atual conceito da preparação física de atletas de alto rendimento preconiza o princípio da especificidade como um dos pontos norteadores na programação, organização e controle das cargas de trabalho específico. Tais medidas são descritas pelos cientistas do desporto, como sendo de grande importância para o sucesso competitivo dos atletas de elite (MATVEEV, 1996; ZAKHAROV, 1992; VERKOSHANSKY, 1990; VERKOSHANSKY, 1998; BOMPA, 1999; OZOLIN, 1989).

A utilização de cargas específicas aplicadas ao judô deve estar em acordo com as informações que possam auxiliar na planificação de treino do judoca, mas ainda sim, esses dados são diversos quanto à sua aplicabilidade metodológica, como pode ser observado nos trabalhos de Franchini et al. (1998, 1999, 2001), Thomas et al. (1989), Callister et al. (1990), Callister et al. (1991), Leplanquais et al. (1994), Pulkinnen (1999), Sterkowicz e Maslej (1998) e Sterkowicz e Franchini (2000), onde a principal preocupação dos autores foi em procurar indicar os tipos de fontes energéticas, suas implicações nos processos fisiológicos e possíveis relações com o overtraining, e ainda, com o aumento da eficácia do processo de preparação do judoca. As primeiras tentativas de se relatar os parâmetros das dinâmicas de luta em judocas brasileiros vêm dos estudos de Rosa (2000), com judocas juniores, e Silva (2002), com lutadores seniores de nível nacional. Outros dados publicados que contribuem dentro desse aspecto são encontrados em citações de Franchini et al. (1999) e trabalhos de Sterkowicz e Maslej, 1998; Castarlenas e Planas 1997; Monteiro, 1995 e Sikorski et al., 1987, sendo que esses dados foram coletados com atletas oriundos de outros países, e que serviram de ponto inicial para os estudos dentro desse assunto no âmbito nacional.

Tal explanação se resume pelo fato de que o controle quantitativo e qualitativo das entradas de golpes, horas de trabalho específico, seja de complexos de treinamento físico ou técnico-tático, não possui uma confiabilidade determinada, visto que não há um número de publicações científicas direcionadas especificamente para esse tipo de ação motora desportiva, que atualmente, é de extrema importância para o processo de preparação de alto rendimento. $O$ desporto contemporâneo necessita desse tipo de informações, pois, sem eles, não é possível relacionar os esforços competitivos, com os meios e métodos apropriados para a otimização do rendimento dos lutadores.

O desenvolvimento contemporâneo do judô, dentro do aspecto competitivo de alto nível, mostra que as necessidades de estudo sobre a sua estrutura e as mudanças que se sucedem ao longo do tempo, são 
determinantes na construção de uma programação do treinamento de judocas de alto nível. As alterações nas regras do judô ocorreram em caráter experimental no ano de 2002 pela Federação Internacional de Judô (IJF, em inglês, International Judô Federation), durante a realização do Campeonato Mundial Junior, na Coréia, sendo que a inclusão do golden score (GS) foi a mais importante; logo após a verificação de sua validade, foi efetivado como nova regra em 2003. Isso modifica o tempo total possível do combate, que passaria de 5 minutos, podendo chegar a até 10 minutos, ou o tempo somado de outra luta. Sendo assim, tais fatos evidenciam uma necessidade de revisão do problema da racionalização dos modos de preparação do treinamento dos judocas, pois o judô, sendo um esporte tradicional concebido no Brasil, geralmente, por meio de difusão cultural, não dispõe de informações conclusivas acerca dos esforços competitivos, e possivelmente, o surgimento esporádico de talentos desportivos tem relação com o desenvolvimento empírico do treinamento de judô, que poderá ser determinante no sucesso competitivo dos atletas de elite (ROSA et al., 2003).

A inclusão da regra do GS tem por objetivo proporcionar outro combate, na seqüência da luta que termina em igualdade de pontos, para que se determine um vencedor sem a necessidade de se decidir pelo modo tradicional, o hantei, ou decisão dos árbitros, tornando o combate de judô mais disputado, pois o judoca conquistando a menor pontuação, ou mesmo uma falta sendo apontada para o seu adversário, encerraria o combate. Finalizado o GS em nova igualdade, os árbitros então são solicitados para a declaração de um vencedor pelo modelo já utilizado. Porém, o critério técnico dos árbitros para a decisão final deverá estar voltado somente ao GS, pois as ações e ataques produzidos na luta anterior não serão avaliados (INTERNATIONAL, 2003).

À luz da teoria do treinamento desportivo, é evidente que as atualizações acerca da referida regra - GS conduzem ao aumento do volume de trabalho competitivo. Isso influenciará diretamente na prescrição do treinamento, que não poderá ser desenvolvida sem conhecimentos prévios de outras ciências ligadas ao rendimento humano. De acordo com Verkoshansky (1990), a preparação dos atletas de alto nível está relacionada, sobretudo, a grandes estímulos dos sistemas funcionais vitais para o organismo, sendo que, sem domínio científico, não é viável solucionar racionalmente os complexos problemas do treinamento moderno do judô, como por exemplo, o controle da intensidade de esforço dos judocas durante uma sessão de treino. 
Com a incorporação dessa regra, acredita-se que o modo como os judocas se preparam deveria ser de maneira diferenciada, atendendo ao Princípio da Especificidade, descrito por Zatsiorsky (1992). Por ocorrer um novo combate, os níveis de resistência específica podem ser mais exigidos, e por isso, deveriam ser condizentes com o tempo em que os atletas estarão em trabalho competitivo. A proposta desse estudo vem ao encontro dessa nova realidade no judô, caracterizada pelo aumento do gasto energético, volume de trabalho específico e tempo de recuperação durante o combate, e espera-se que possa vir a responder tais questionamentos na preparação física de judocas de alto nível.

\section{OBJETIVOS}

estabelecer parâmetros temporais da dinâmica da luta de judocas brasileiros;

predizer possíveis implicações do GS na estruturação do processo de treinamento específico aplicado aos judocas, baseado na quantificação do tempo de esforço competitivo.

\section{METODOLOGIA}

Tipificação do estudo: caracteriza-se como pesquisa observacional, quantitativa de retro-análise (ROUQUAYROL; ALMEIDA FILHO, 1999).

Grupo de estudo: 23 judocas do sexo masculino, de todas as categorias de peso. Os atletas são filiados à Confederação Brasileira de Judô (CBJ), que se responsabilizou pela organização do sistema de disputa da $1^{\text {a }}$ Seletiva Nacional de Judô de 2003, realizada na cidade de São Paulo, no dia $1^{\circ}$ de fevereiro. Para essa competição, os participantes foram selecionados, em cada categoria, a partir dos seguintes eventos: vencedor do Campeonato Brasileiro de 2002, vencedor do Troféu Brasil Interclubes de 2002, $2^{\circ}$ colocado do ranking nacional em 2002, e somente nas categorias ligeiro e médio, foi indicado um atleta pela comissão técnica da CBJ. O vencedor disputou uma seletiva final com o $1^{\circ}$ colocado do ranking nacional em 2002, assim, os vencedores desta última disputa formaram a seleção nacional que participou do Campeonato Pan-Americano de Judô, Jogos Pan-Americanos e Campeonato Mundial de Judô, realizadas no ano de 2003.

Coleta dos dados: as filmagens foram realizadas no shiai-jô montado com duas áreas oficiais (INTERNATIONAL, 2003), nas dependências do ginásio poliesportivo do Esporte Clube 
Pinheiros, sob a autorização da Comissão Técnica da CBJ. Com a utilização de duas câmeras

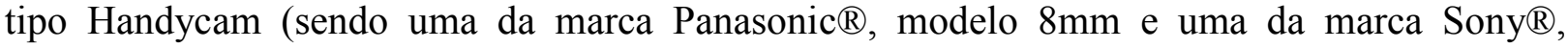
modelo VHS-C), as filmagens das lutas dos atletas foram feitas na sua totalidade (desde o momento de entrada, até a saída dos judocas do local de competição), onde toda a área de combate era focalizada, a uma distância aproximada de cinco metros da borda mais próxima do shiai-jô até o local da câmera filmadora. No total, de todas as categorias de peso, foram filmadas 42 lutas.

Análise dos dados: as 42 lutas foram analisadas, levando-se em conta os seguintes parâmetros, em segundos:

$\checkmark \quad$ Tempo de luta (TL): tempo de luta ou período entre a voz de comando do árbitro para iniciar o combate (hajimê), e a voz de comando para parar o combate (mattê, para interromper; sore madê, para terminar a luta). É necessário ressaltar que o tempo de luta no solo foi computado nesse item, pois se trata de ação motora competitiva, sendo posteriormente avaliado como um item à parte.

$\checkmark \quad$ Tempo de recuperação (TR): o tempo de recuperação compreendeu o período entre o sinal de interrupção do combate (voz de comando mattê), e o sinal de reinício do combate (voz de comando hajimê). Os comandos sono mama e yoshi (interrupção e prosseguimento do combate no solo) também foram alvo da análise.

Tempo de luta no solo (TLS): o tempo de luta no solo compreendeu o tempo onde um ou ambos os atletas caracterizaram o trabalho de nê waza (técnica de solo), previsto na regra. Atendimento médico (AM): dentro desse estudo, atendimento médico seriam todas as pausas de recuperação que foram contadas como item em separado do TR, pois são valores freqüentemente altos que ultrapassam 60 segundos de duração. Incluem-se também as pausas para troca de kimono, que se rasgou durante lutas da Seletiva, sendo situações em que os judocas param de lutar por mais tempo, casos estes previstos na regra (INTERNATIONAL, 2003).

Com a análise das lutas realizadas dentro dessas variáveis, os dados foram separados para uma nova análise em dois grupos: grupo 1 (G1), os dados analisados dos combates sem sistema de GS (sendo TL, TR, TLS e AM); e Grupo 2 (G2), dados das lutas com o sistema de GS (sendo TLGS, o item "a" como 
parte da luta com GS; TRGS, o item "b" dentro do GS; TLSGS, o item "c" no GS; AMGS, o item "d" no GS). Das 42 lutas analisadas - dez foram decididas no GS, sendo que quatro terminaram por conquista de ponto, três por falta cometida, e três por hantei. Todos os itens foram avaliados dentro das regras oficiais da IJF, para que houvessem padronizações dos resultados obtidos.

Para a análise estatística, calcularam-se médias e desvio-padrão, sendo a comparação entre os grupos de dados feitos pelo teste ANOVA para valores não paramétricos (teste Dunn de comparação múltipla).

\section{RESULTADOS E DISCUSSÕES}

Os resultados obtidos pelos atletas, para os itens descritos, durante o desenvolvimento das lutas, são mostrados em média e desvio-padrão respectivamente, na Tabela 1. Os números entre parêntesis representam, em segundos, os valores mínimos e máximos das intervenções no combate em cada item. Os dados acerca do desempenho competitivo dos atletas da Seletiva Nacional não apresentaram diferenças significantes $(p<0,05)$, por meio de comparação entre os dois grupos.

\section{TABELA 1 - MÉDIA E DESVIO-PADRÃo DAS VARIÁVEIS ANALISADAS DENTRO DA SITUAÇ̃̃o COMPETITIVA, EM SEGUNDOS $(\mathbf{P}<0,05)$}

\begin{tabular}{ccc}
\hline $\begin{array}{c}\text { VARIÁVEL DA } \\
\text { LUTA }\end{array}$ & \multicolumn{2}{c}{ GRUPO DE DADOS DA LUTA } \\
& Grupo $1(\mathrm{sem}$ GS, $\mathrm{n}=32$ lutas $)$ & Grupo $2($ com GS, $\mathrm{n}=10$ lutas $)$ \\
TL & $30,41 \pm 20,69(2-165)$ & $28,40 \pm 17,80(4-89)$ \\
\hline TR & $7,41 \pm 5,19(3-46)$ & $8,04 \pm 5,99(3-37)$ \\
TLS & $9,51 \pm 7,28(3-44)$ & $13,00 \pm 9,78(4-29)$ \\
AM & $74,68 \pm 23,03(48-133)$ & $76,34 \pm 14,25(56-95)$ \\
\hline
\end{tabular}

Os resultados médios obtidos nas variáveis acima descritas do Grupo 1 indicam que os períodos de intervenção de combate estão próximos de 30 segundos, com pausas de, em média, 7 segundos e luta no solo em torno de 9 segundos. Já o Grupo 2 apresentou menor valor médio para TL (28s), e valores médios mais elevados para TR, TLS e $\operatorname{AM}(8,13$ e 76 segundos, respectivamente). Isto implica que o direcionamento dos meios específicos de treinamento deve permear esses valores, considerando suas respectivas amplitudes.

Os valores de cada período de ocorrência dos Grupos 1 e 2 estão dispostos, respectivamente, nos Gráficos 1 e 2; os tempos de AM também aparecem dentro dessa descrição. De acordo com o histograma dos dados de cada grupo, é possível observar que os dados fogem do padrão da curva de normalidade, como parece ser característico dos desportos de combate, pois não há um padrão previsível dentro das lutas. 


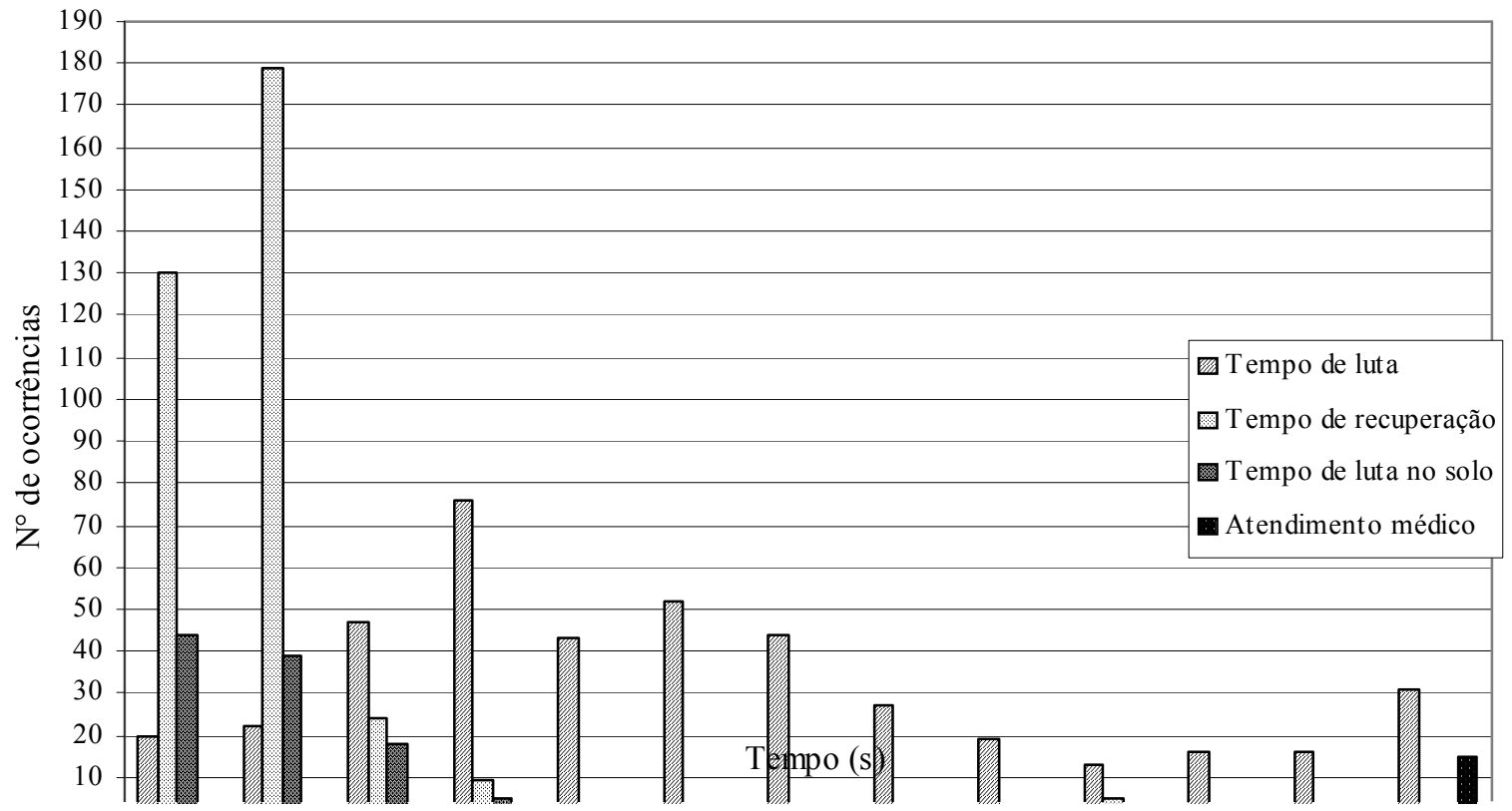

Gráfico 1 - Histograma do tempo (em segundos) das intervenções do grupo 1, dentro dos combates dos atletas da seletiva nacional

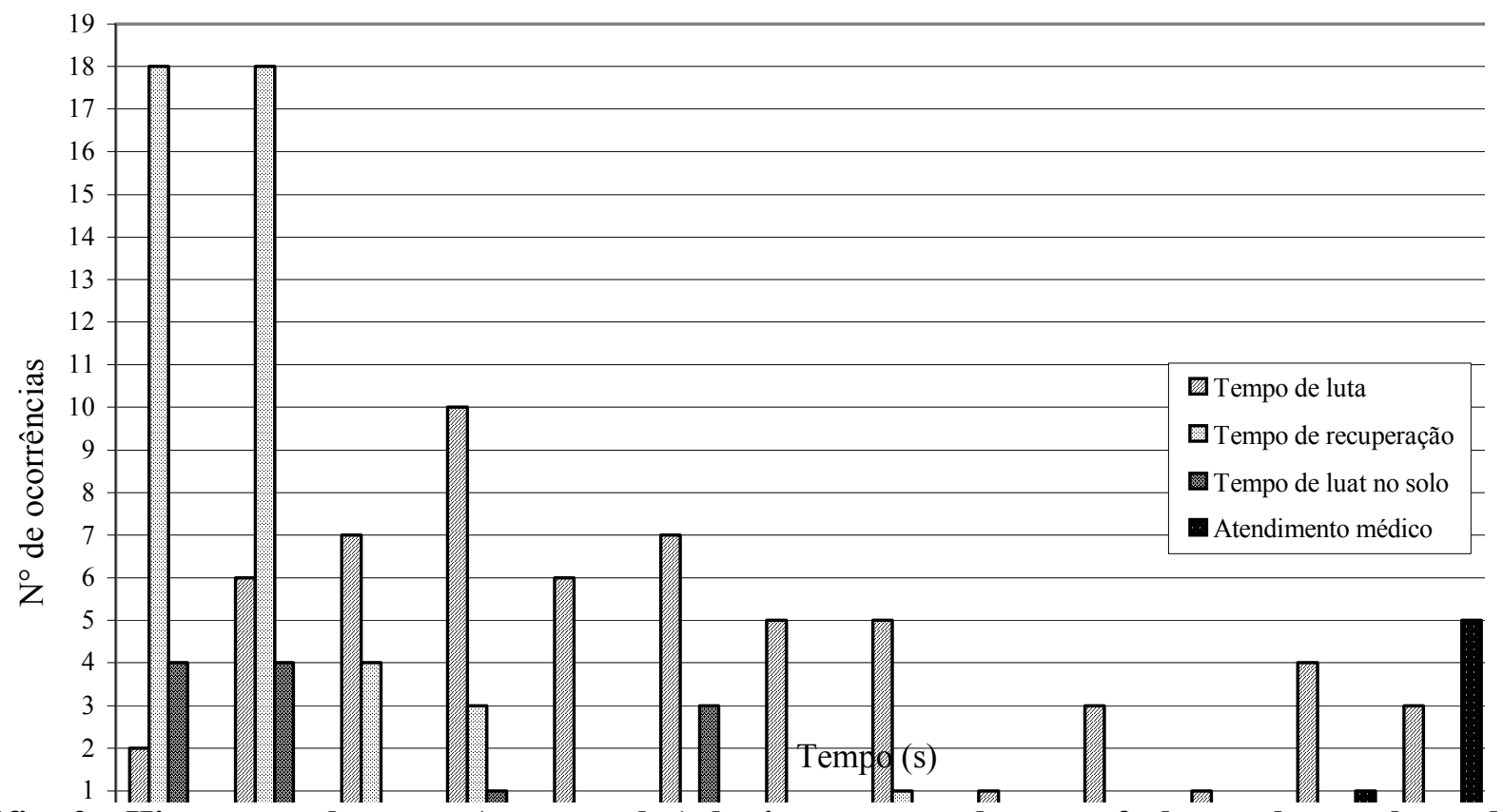

Gráfico 2 - Histograma do tempo (em segundos) das intervenções do grupo 2, dentro dos combates dos atletas da seletiva nacional 
Os dados descritos acima podem indicar o caráter acíclico das intervenções dentro do combate de judô, já que as variações de tempo não têm um controle determinado nas ações, sendo reforçado com as medidas descritivas, como os valores medianos, quartis e extremos mostrados no Gráfico 3 (box-plot).

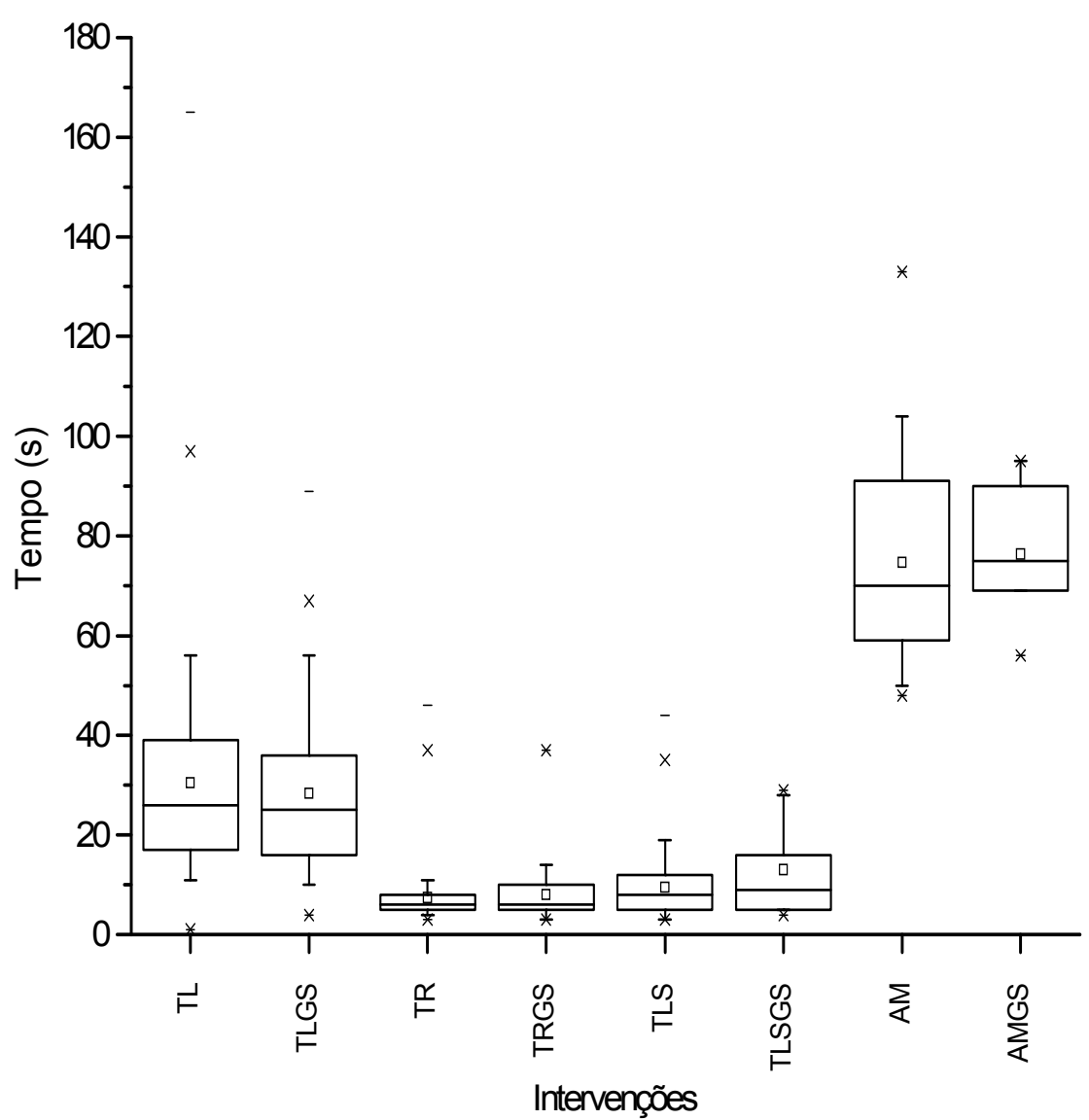

TL: tempo de luta

TLGS: tempo de luta no Golden Score (GS)

TR: Tempo de recuperação TRGS: tempo de recuperação no GS

TLS: tempo de luta no solo TLSGS: tempo de luta no solo no GS

AM: atendimento médico AMGS: atendimento médico no GS

\section{Gráfico 3: Medidas descritivas das ocorrências de intervenções dentro dos combates da Seletiva Nacional (em segundos)}

O advento desta nova regra exprime uma mudança nos níveis de resistência específica a serem exigidos dos judocas de alto nível. Mesmo não havendo uma diferença significante entre os valores totais das intervenções de luta e dos valores das lutas inseridas na nova regra, é possível observar que pode existir uma relação entre o tempo total de luta, que aumenta conforme a determinação do regulamento, e a dinâmica de esforço mobilizado pelo lutador durante o combate. Sob o aspecto metodológico do treinamento desportivo, sugere-se que pode ocorrer modificação na instalação da fadiga, visto a ocorrência do aumento do volume específico nas lutas com GS. Isso reforça a idéia expressa por Verkoshansky (1990, p. 29), que classifica as lutas como "desportos combinados", pois possuem grande 
variabilidade de movimentação, esforço físico intermitente de elevada intensidade, com pausas de curta duração durante as lutas, que nesse estudo, ocorreram relativamente, com maior freqüência, próximo a 10 segundos. Tal valor tem proximidade com os achados de Rosa (2000), onde as médias estão em torno de 11 segundos, e corrobora com dados de Sterkowicz e Maslej (1998), onde os valores médios correspondem a 10 segundos. É necessário ressaltar que as diferenças desses valores podem ter relação com as regras vigentes na coleta de dados de cada trabalho, pois isso influencia de modo determinante nos resultados de análise atuais. O atual panorama do desporto sugere que as lutas apresentam maior magnitude de esforços, se comparados os dados de trabalhos anteriormente citados.

No que é possível dizer sobre o aspecto técnico-tático, as dinâmicas de luta são mantidas, mas se levarmos em conta a recuperação (TR), a luta no solo (TLS) e o atendimento médico (AM), são evidentes as reações dos judocas quando acontece uma passagem para o solo durante o GS.

Tal fato pode indicar uma forma de demonstrar a iniciativa de quem se aproveita de uma situação de ataque, seja em pé ou partindo para a luta no solo. De acordo com a regra (IJF, 2003), as ações de combate consideradas no hantei serão das ocorridas no GS, e devido a essa norma, os judocas procurarão demonstrar ofensividade, tentando converter esse panorama a seu favor caso não ocorra ponto, e com isso, a decisão dos árbitros será criteriosa nesse sentido. E, ao mesmo tempo, deve-se pensar que essas implicações de intensidade elevada de esforço poderiam acarretar aumento das pausas de recuperação, embora tal tendência não foi constatada na presente investigação. O judoca se utiliza desse recurso tático para estender o descanso, que pode ser decisivo no combate, visto os níveis de esforço quantificados pelas concentrações médias de lactato sanguíneo encontradas em atletas, nos períodos pós-lutas, por Franchini et al. (2001), que observaram 11,77 mM; Callister et al. (1990), com 7,80 mM; Callister et al. (1991), que reportaram 8,40 mM, e Thomas et al. (1989), com 14,50 mM.

É necessário ainda considerar que, taticamente, o atleta pode tentar manipular a duração das pausas durante a luta, facilitando o próprio processo de recuperação, quer seja durante a pausa dada pelo árbitro, ou no AM. O judoca de alto nível deve ter consciência que, do mesmo modo que ele pode manipular esse fator, existem outros fatores que não há um controle determinado, como os critérios dos juízes que, por exemplo, podem ter influências do ambiente competitivo, e isso indica o quanto é necessário o judoca estar bem preparado para o evento.

$\mathrm{O}$ andamento da luta pode acarretar ganhos ou perdas numa dependência direta do nível de preparação física, técnica, tática e psicológica do atleta. Por outro lado, deve-se considerar que a adoção de diferentes 
técnicas de luta está relacionada aos diferentes biotipos de lutadores, conforme sugerido por Sterkowicz e Franchini (2000), e da diferenciação morfológica e funcional dos sistemas vitais em função da adaptação à especificidade do esforço dos atletas, conforme sugere Kingue et al. (2001) e Cottin et al. (2001).

Fica evidente a necessidade de se estabelecer o perfil do judoca brasileiro de alto rendimento e através da união teórico-prática, do que se considera em Ciências do Desporto Contemporâneo, possibilite melhorar ainda mais a qualidade do judô nacional.

\section{CONCLUSÕES}

Com base na apresentação dos dados contidos nesse trabalho, podemos citar as seguintes conclusões desse estudo, que seriam:

> Não houve diferença significativa entre os grupos de dados das dinâmicas de combate apresentadas pelos judocas nas lutas sem e com GS;

$>\mathrm{O}$ aumento no volume de esforço competitivo, decorrente da inclusão do GS, tende a evidenciar o estado de fadiga compensada como um dos fatores determinantes de nova estratégia para preparação física em conjunto com o aspecto técnico-tático, visando aumento da resistência específica dos judocas durante os maiores períodos de combate;

$>$ De acordo com os tempos de esforço e recuperação, sugere-se que a participação do sistema anaeróbio aláctico e láctico são importantes para a realização do trabalho competitivo durante as lutas que se prolongam para o GS;

As relações com a planificação de treinamento a serem adotadas, mostram uma necessidade de atenção para o treino da resistência especial de luta com tolerância ao lactato, e da resistência aeróbia, como auxiliar no processo de ressíntese do ATP; 
Reforça-se a necessidade de novos estudos dentro desse tema, na tentativa de aumentar a quantidade e qualidade das informações sobre as manifestações de esforços do judô de competição, para que se permita estabelecer princípios metodológicos sobre a preparação física, técnica e tática de judocas de elite.

\section{REFERÊNCIAS}

BOMPA, T. O. Periodization training for sports. Champaign: Human Kinetics, 1999.

CALLISTER, R. et al. Physiological and performance responses to overtraining in elite judo athletes. Medicine and Science in Sports and Exercise, v. 22, n. 6, p. 816-824, 1990.

CALLISTER, R. et al. Physiological characteristics of elite judo athletes. International Journal of Sports Medicine, v. 12, p. 196-203, 1991.

COTTIN, F. et al. Étude comparative de l'analyse spectrale de la fréquence cardiaque au cours de l'exercice sur ergocycle et de l'entraînement en judo (Heart rate comparative study by spectral analysis between two exercises: ergocycle vs judo randori). Science \& Sports, v. 16, n. 6, p. 295-305, 2001.

FRANCHINI, E. et al. Características fisiológicas em testes laboratoriais e resposta da concentração de lactato sangüineo em 3 lutas em judocas das classes Juvenil, Junior e Sênior. Revista Paulista de Educação Física, São Paulo, v. 12, n. 1, p. 5-16, 1998.

. Análise de um teste específico para o judô. Kinesis, Santa Maria, n. 21, p. 91-108, 1999.

- Efeito do tipo de recuperação após uma luta de judô sobre o lactato sanguíneo e sobre o desempenho anaeróbio. Corpoconsciência, São Paulo, n. 7, p. 23-39, 2001.

INTERNATIONAL Judô Federation (IJF). Atualização e interpretação das novas regras. Disponível em: <http://www.ijf.org>. Acesso em: 2003.

KINGUE, S. et al. Étude échographique de la fonction ventriculaire gauche d'un groupe de judokas camerounais (Ultrasonographic study of left ventricular function in a group of cameroonian judokas). Science \& Sports, v.16, n.1, p.10-15, 2001.

LEPLANQUAIS, F. et al. Propositions pour une musculation spécifique: exemple du judo. Cinésiologie, n. 160 , p. 80-86, 1994.

MATVEEV, L. P. Preparação desportiva. Londrina, Centro de Informações Desportivas, 1996.

PULKINNEN, W. Anaerobic requirements of elite judo athletes. Disponível em: Site: $<$ http://www.judoinfo.com>. Acesso em: 1999. 
ROSA, R. R. Estudo da quantificação das ações motoras e esforços específicos de atletas de judô em situação competitiva. 2000. 75F. Monografia (Graduação) - Faculdade de Educação Física, Universidade Estadual de Campinas, Campinas, 2000.

ROSA, R. R.; DEL VECCHIO, F. B.; OLIVEIRA, P. R. Estudo quantitativo da dinâmica da luta de judocas brasileiros. In: SIMPÓSIO INTERNACIONAL DE CIÊNCIAS DO ESPORTE, 26., São Paulo, 23 a 25 de outubro. Anais... São Paulo: CBCE, 2003. p. 45.

ROUQUAYROL, M. Z.; ALMEIDA FILHO, N. Epidemiologia \& saúde. Rio de Janeiro: Medsi, 1999.

SILVA, W. N. C. Judô: estudo das ações motoras especificas da competição - Estratégias metodológicas para o treinamento. 2002. 82 f. Dissertação (Mestrado) - Faculdade de Educação Física, Universidade Estadual de Campinas, Campinas, 2002.

STERKOWICZ, S.; MASLEJ, P. An evaluation of modern tendencies in solving judo fights. Disponível em: <http://www.judoinfo.com>. Acesso em: 1998.

STERKOWICZ, S.; FRANCHINI, E. Techniques used by judoists during the World and Olympic Tournaments 1995-1999. Human Movement, v. 2, n. 2, p. 24-33, 2000.

THOMAS, S. G. et al. Physiological profiles of the Canadian judo team. Canadian Journal of Sports Sciences, n. 3, p. 142-147, 1989.

VERKOSHANSKY, Y. V. Entrenamiento deportivo: planificacion e programacion. Barcelona: Martinez Roca, 1990.

OZOLIN, N. G. Sistema contemporaneo de entrenamiento deportivo. 3. ed. Havana: Ed. Científico Tecnico, 1989.

ZAKHAROV, A. Ciência do treinamento desportivo. Rio de Janeiro: Grupo Palestra Sport, 1992.

ZATSIORSKY, V. Ciência e prática do treinamento de força. São Paulo: Phorte, 1999. 


\section{Rodrigo Ribeiro Rosa}

Faculdade de Educação Física/UNICAMP

\section{Fabrício Boscolo Del Vecchio}

Faculdade de Educação Física/UNICAMP

\section{Leonardo José Mataruna dos Santos}

Faculdade de Educação Física/UNICAMP

\section{Mara Patrícia Traina Chacon-Mikahil}

Faculdade de Educação Física/UNICAMP

\section{Paulo Roberto de Oliveira}

Faculdade de Educação Física/UNICAMP

\section{Referência do artigo:}

\section{ABNT}

ROSA, R. R. et. al. Estudo da dinâmica da luta e a influência do golden score na preparação física de judocas de alto nível. Conexões, v. 6, n. 1, p. 40-53.

\section{APA}

Rosa, R. R., Vecchio, F. B., Santos, L. J. M., Chacon-Mikahil, M. P. T., \& Oliveira, P. R. (2008). Estudo da dinâmica da luta e a influência do golden score na preparação física de judocas de alto nível. Conexões, 6(1), 40-53.

\section{VANCOUVER}

Rosa RR, Vecchio FB, Santos LJM, Chacon-Mikahil MPT, Oliveira PR. Estudo da dinâmica da luta e a influência do golden score na preparação física de judocas de alto nível. Conexões, 2008, 6(1): 40-53. 